\title{
BreastCore
}

\section{Radiotherapy of Ductal Carcinoma In Situ}

\author{
David Krug $^{\text {a }}$ Rainer Souchon ${ }^{b}$ \\ ${ }^{a}$ Radioonkologie und Strahlentherapie, Universitätsklinikum Heidelberg, Germany; \\ ${ }^{b}$ Formerly University Hospital Tübingen, Germany
}

\author{
Keywords \\ Ductal carcinoma in situ (DCIS) - Radiotherapy . \\ Low-risk subgroup · Boost · Hypofractionation
}

\section{Summary}

Ductal carcinoma in situ (DCIS) is a heterogeneous disease in both its biology and clinical course. In the past, recurrence rates after breast-conserving surgery have been as high as $30 \%$ after 10 years. The introduction of mammography screening and advances in imaging have led to an increase in the detection of DCIS. The focus of this review is on the role of radiotherapy in the multidisciplinary treatment, including current developments in hypofractionation and boost delivery, and attempts to define low-risk subsets of DCIS for which the need for adjuvant radiation is repeatedly questioned.

(c) 2015 S. Karger GmbH, Freiburg

\section{Introduction}

Pure ductal carcinoma in situ (DCIS) accounts for about $16 \%$ of all screening-detected malignancies of the breast [1]. It represents a biologically heterogeneous group of non-invasive lesions in the breast and should be termed more precisely ductal intraepithelial neoplasia (DIN) with regards to its non-malignant behavior [2] Nevertheless, DCIS has a potential for progression to invasive carcinoma, which characterizes DCIS as a pre-invasive or precursor lesion. DCIS is considered to be a significant risk factor for local relapse (LR) after breast-conserving surgery (BCS). LR may occur as pure DCIS or, in about $50 \%$ of cases, as invasive breast cancer. Rates of LR of DCIS are high and may exceed those for invasive cancer after BCS. This fact strongly supports recommendations for relapse-reducing strategies after BCS for pure DCIS aimed at organ preservation and an alternative to mastectomy.

Adjuvant radiotherapy (RT) has proven to be effective in the prevention of LR after BCS [3], but has no impact on survival rate within a 20-year follow-up period [4]. Since the risk of subsequent death due to breast cancer after treatment for DCIS is negligible, much effort has been directed towards the identification of women considered at low risk of recurrence in whom additional treatment could safely be omitted.

In patients receiving $\mathrm{RT}$, hypofractionation might offer an alternative, more convenient option with lower associated healthcare costs [5]. Application of a tumor bed boost significantly lowers the risk of LR in invasive breast cancer [6], while its role in DCIS remains unclear.

\section{Identification of Patients at Low Risk of Recurrence}

Four randomized controlled trials (RCTs) have shown the efficacy of RT in reducing the risk of LR in patients with DCIS after BCS. A detailed discussion of these individual trials is beyond the scope of this review. In the 2010 meta-analysis of the Early Breast Cancer Trialists' Collaboration Group [3], the relative risk reduction was $54 \%$ (rate ratio $0.46 \mathrm{p}<0.0001$ ). The absolute benefit from RT, however, strongly depends on the individual risk of recurrence. Furthermore, although there was a highly significant improvement in local control, none of the trials demonstrated any benefit of RT in terms of distant metastases or survival. This was recently confirmed by the 20-year data of the SweDCIS trials [4]. Of 1,067 patients undergoing randomization, only 41 died due to breast cancer $(3.8 \%)$.

Thus, many researchers have tried to identify subgroups of patients with a presumably low risk of recurrence in whom RT could safely be omitted. Table 1 gives an overview over the different classifications included in the publications, and these are described in detail here.

\section{KARGER}

Fax +497614520714

\section{(c) 2015 S. Karger GmbH, Freiburg}

$1661-3791 / 15 / 0104-0259 \$ 39.50 / 0$
Dr. med. David Krug 
Table 1. Overview of parameters included to identify a subset of low-risk DCIS patients and corresponding LR rates without $\mathrm{RT}$

\begin{tabular}{|c|c|c|c|c|c|}
\hline Trial & Patients, $\mathrm{n}$ & Timeframe & Included prognostic factors & $\begin{array}{l}\text { Corresponding LR rates } \\
\text { without RT, \% (period) }\end{array}$ & $\begin{array}{l}\text { Use of } \\
\text { tamoxifen, \% }\end{array}$ \\
\hline ECOG E5194 [15] & $\begin{array}{l}565 \\
105\end{array}$ & 1997-2002 & $\begin{array}{l}\text { grading G1-2 and } \\
\text { tumor size } \leq 2.5 \mathrm{~cm} \\
\text { grading G3 and tumor size } \\
\leq 1 \mathrm{~cm} \text {; margins } \geq 3 \mathrm{~mm}\end{array}$ & $\begin{array}{l}10.5(7 \mathrm{y}) \\
18(7 \mathrm{y})\end{array}$ & 30 \\
\hline DF/HCC $[16,17]$ & 158 & 1995-2002 & $\begin{array}{l}\text { grading G1-2 and } \\
\text { tumor size } \leq 2.5 \mathrm{~cm} \text { and } \\
\text { margins } \geq 10 \mathrm{~mm}\end{array}$ & $12(5 \mathrm{y})$ & n.s. \\
\hline RTOG 9804 [20] & 636 & 1998-2006 & $\begin{array}{l}\text { grading G1-2, } \\
\text { tumor size } \leq 2.5 \mathrm{~cm}, \\
\text { margins } \geq 3 \mathrm{~mm}\end{array}$ & $6.7(7 \mathrm{y})$ & 62 \\
\hline $\begin{array}{l}\text { VNPI [8] } \\
\qquad \begin{array}{l}3-4 \\
5-7 \\
8-9\end{array}\end{array}$ & 333 & 1979-1995 & $\begin{array}{l}\text { Tumor size, } \\
\text { margin width, } \\
\text { pathological classification }\end{array}$ & $\begin{array}{r}3(8 \mathrm{y}) \\
32(8 \mathrm{y}) \\
100(8 \mathrm{y})\end{array}$ & n.s. \\
\hline $\begin{array}{l}\text { DCIS score }[14]^{\mathrm{a}} \\
\text { Low risk } \\
\text { Intermediate risk } \\
\text { High risk }\end{array}$ & 327 & 1997-2002 & 12 genes from Oncotype Dx & $\begin{array}{l}10.6 \\
26.7 \\
25.9\end{array}$ & 29 \\
\hline
\end{tabular}

The Van Nuys classification discriminates 3 risk categories by the presence of comedonecrosis and the histological grading [7]. In the seminal publication, LRs occurred in $3.8 \%, 11.1 \%$ and $26.5 \%$ of patients in the 3 groups after BCS with or without RT [7]. The classification has been modified to include margin width, resulting in the Van Nuys Prognostic Index (VNPI, [8]), and by adding patient age, yielding the University of Southern California (USC)/VNPI [9]. Results from a cohort of 706 patients with DCIS suggested that the USC/VNPI could be used to stratify patients according to their recurrence risk to guide treatment decisions. Low-risk patients did not significantly benefit from RT, whereas high-risk patients had high recurrence rates despite RT, possibly making them candidates for mastectomy [9]. Several researchers have since tried independently to validate the VNPI, but not all were successful [10, 11]. So far, there has been no prospective validation of the VNPI or the USC/VNPI.

There have been attempts to improve the VNPI by adding molecular information to the histopathological parameters. Altintas et al. [12] showed that the genomic grade index, a 97-gene signature, might improve the accuracy of the VNPI in identifying high-risk patients. However, these results were based on a retrospective review of 88 cases with 10 events.

There have been other attempts to integrate molecular biology into risk prediction for DCIS. Kerlikowske et al. [13] performed immunohistochemistry for various biomarkers in a nested casecontrol study of 324 patients. They showed that the risk of invasive recurrence was linked to the presence of a p16, COX-2, and Ki67 triple-positive immunophenotype, whereas non-invasive recurrence was increased in patients with either $\mathrm{ER}^{-} / \mathrm{ERBB}^{+} / \mathrm{Ki} 67^{+}$or p $16^{+} / \mathrm{COX}-2^{-} / \mathrm{Ki}^{+} 7^{+}$status.
Solin et al. [14] used the OncotypeDX test to establish a DCIS score that was independently validated in a subset of patients from the ECOG E5194 study [15]. The DCIS score was an independent predictor of LR on multivariate analysis (hazard ratio (HR) 2.37, $\mathrm{p}=0.02$ ). The 10 -year LR was $10.6 \%, 26.7 \%$, and $25.9 \%$ in 3 prespecified risk groups (low, intermediate and high risk, respectively). Invasive recurrences occurred in 3.7\%, 12.3\%, and 19.2\%, of patients, respectively $(\mathrm{p}=0.003)$.

The ECOG E5194 trial [15] was a prospective study of patients with presumed low-risk DCIS who received BCS without RT. 565 patients with low- or intermediate-grade DCIS measuring $\leq 2.5 \mathrm{~cm}$ and 105 patients with high-grade DCIS measuring $\leq 1 \mathrm{~cm}$ were enrolled. Recruitment into the high-grade arm was stopped prematurely due to slow accrual. The minimum margin width was $3 \mathrm{~mm}$. Approximately $30 \%$ of patients received tamoxifen. The 5- and 7-year LR rates were $6.1 \%$ and $10.5 \%$ in the lowand intermediate-grade group and $15.3 \%$ and $18 \%$ in the highgrade group, respectively.

A similar prospective phase II trial in Boston [16] was stopped early when the stopping boundary for LR was reached after accrual of 158 patients. Patients with low/intermediate-grade DICS of $\leq 2.5 \mathrm{~cm}$ and margins of $\geq 1 \mathrm{~cm}$ were included; the use of tamoxifen was not permitted. The LR rate was $2.4 \%$ per patient year. In a subsequent publication, LR after 8 years was $13 \%$, and the 10 -year LR rate was estimated to be $15.6 \%$ [17].

A recent publication of pooled data for 314 women from 5 prospective trials suggested that molecular subtypes could be predictors of LR in DCIS [18]. After 5 years, LR were $7.6 \%$ for luminal A-type DCIS, 23.2\% for luminal B-type, 36.1\% for HER2-type and $15.8 \%$ for triple-negative DCIS. High Ki67 expression and molecu- 
lar subtype were independent prognostic factors in multivariate analysis. However, these data have to be interpreted with caution since $65 \%$ of patients had high-grade DCIS. Only $66 \%$ of patients had BCS, with $14-19 \%$ having adjuvant RT.

Another study performed in Milan, including 1,667 patients, analyzed the role of HER2 expression as a prognostic factor in DCIS [19]. The risk of non-invasive recurrence was increased in patients with HER2 overexpression, while there was no difference for invasive recurrences. Interestingly, the increase in non-invasive recurrences was mainly driven by HER2-positive patients not receiving adjuvant RT.

In conclusion, many groups have tried to establish clinical, histopathologial and molecular parameters to identify patients with DCIS at low risk of LR in whom RT could safely be omitted. However, many different parameters were used and validation in independent retrospective cohorts is often lacking. Thus, no uniform definition of a low-risk subset exists.

\section{Results of RT in Low-Risk DCIS: The RTOG 9804 Trial}

The results of the prospective randomized RTOG 9804 phase III trial have recently been published [20]. In this protocol, patients with low-risk DCIS (defined as a unifocal tumor of $<2.5 \mathrm{~cm}$, low or intermediate nuclear grading and margin width of $\geq 3 \mathrm{~mm}$ ) were randomized to postoperative normo-fractionated whole-breast RT of 50-50.4 Gy administered in 25-28 fractions after BCS or observation. Tamoxifen was required during the first few years of the trial. In 2001, an amendment was added to make the use of tamoxifen optional and to allow hypofractionation of RT with $42.5 \mathrm{~Gy}$ in 16 fractions. The study was terminated before meeting its target accrual due to slow recruitment. The LR rates after 5 and 7 years were $0.4 \%$ and $0.9 \%$ for patients treated with RT and $3.5 \%$ and $6.7 \%$ for patients treated without RT (HR 0.11, p $<0.001$ ). Patients assigned to RT had a higher rate of grade 1-2 acute toxicity, but the rates of acute and late grade 3 toxicity were low, and there was no grade 4-5 toxicity. As expected, there was no difference in overall survival in the study arms.

The authors concluded that, using their trial inclusion criteria, a low-risk subset of DCIS patients has been successfully identified. As remarkable main result of this trial, it was clearly demonstrated the addition of RT significantly decreased the LR rate even in these favorable risk patients. However, the rise in the LR rates from 5 to 7 years is certainly of concern and is in line with other publications showing a steady increase in the risk of LR also after 5 years $[3,4]$. Thus, longer follow-up is necessary to estimate the true impact of the omission of RT in these patients. One should also keep in mind that the use of tamoxifen (especially in low-risk DCIS) is not a standard procedure in many countries and that the risk of LR might be higher in this setting.

Notwithstanding, patients with a low-risk profile (according to the RTOG 9804 patient characteristics) should receive counseling about the potential benefits and risks of RT. This should also in- clude the fact that the risk of severe late adverse effects due to RT is low nowadays if RT is performed according to guideline recommendations $[2,21,22]$. A recent population-based study from the Netherlands found no increased risk for cardiovascular morbidity or mortality in patients with DCIS treated with postoperative RT [23].

\section{Application of a Tumor Bed Boost in DCIS}

The application of a boost to the lumpectomy cavity in addition to whole-breast irradiation after BCS significantly reduces LR rates in invasive breast cancer with the highest benefit for younger patients $[6,21]$. In the large randomized trials included in the EBCTCG meta-analysis [3], boost irradiation was only used in a minority of DCIS patients. Several publications have addressed the role of a boost in patients with DCIS, especially those of young age or with other adverse prognostic factors. All of these studies are retrospective comparisons, most of them from single institutions. Patients treated with a boost usually had more risk factors for LR, especially close or positive margins. Furthermore, large variations exist between the studies due to the geographical and temporal differences.

Julian et al. [24] presented an abstract of an analysis from the NSABP B24 trial analyzing the impact of an additional boost in a subgroup of 1,569 patients. While risk factors such as positive resection margins and comedonecrosis were more common in patients receiving a boost, there was no difference in LR (HR 1.12, $\mathrm{p}=0.69$ ). The largest series so far was a population-based analysis from Ontario with 1,895 patients. The 10-year LR rate was $13 \%$ without a boost and $12 \%$ with a boost $(\mathrm{p}=0.27)$.

There are some studies suggesting that a reduction of LR can be achieved by application of a boost to the lumpectomy cavity. A multicenter retrospective study of 373 patients from the Rare Cancer Network was published in 2006 [25]. The median age was 41 years; $7 \%$ of patients received tamoxifen. Results showed that the addition of a tumor bed boost significantly reduced LR (HR 0.45, $\mathrm{p}=0.024$ ). However, bias cannot be excluded since information on baseline prognostic factors were missing for many patients and because patients were treated over a period of 26 years.

Wong et al. [26] performed a retrospective analysis of 220 patients treated at McGill University. Although positive margins were observed more frequently and the VNPI showed a higher baseline recurrence risk, application of a boost significantly reduced LR $(p=0.03)$. No LR was observed in 79 patients receiving a boost after a median follow-up of 46 months.

Researchers from the University of Florence published their findings after delivering a boost to 389 patients [27]. Again, close or positive margins and a higher VNPI were more common in the patients receiving a boost, and both parameters were associated with a significantly higher risk of LR on univariate analysis. No significant relationship between LR and boost delivery was found on univariate analysis (HR $0.5, \mathrm{p}=0.086$ ), but on multivariate analysis it emerged as an independent prognostic factor (HR 0.17, $\mathrm{p}=0.014$ ). 
Monteau et al. [28] suggested that boost irradiation could present an alternative to re-excision in patients with close $(<2 \mathrm{~mm})$, focally $(<1 \mathrm{~mm})$ or minimally $(1-15 \mathrm{~mm})$ involved margins. In their analysis of 208 patients, 61 patients had a re-excision with $56 \%$ having residual non-invasive disease. A boost was used in $58 \%$ of patients with and $92 \%$ of patients without re-operation. The 7 -year LR rate was $9.3 \%$ vs. 9.6\% ( $\mathrm{p}=0.93$ ).

Nilsson et al. [29] summarized the available studies in a recently published meta-analysis of 12 retrospective studies with a total of 6,943 patients. The overall quality of the included studies was rated as very low. There was no statistically significant advantage of an additional boost over whole-breast irradiation alone after BCS (HR $0.91, \mathrm{p}=0.47)$. However, if only patients with positive margins were included, there was a significant benefit (odds ratio 0.56 , $\mathrm{p}=0.01)$. The authors also performed subgroup analyses according to patient age ( $<50$ and $<45$ years), but no significant relationship was found.

Taken together, the addition of a boost to the lumpectomy cavity after whole-breast irradiation might reduce LR rates in patients at high risk, especially those with positive margins. However, the validity is greatly hampered by the low quality of evidence.

Currently, there are 2 prospective randomized trials addressing the role of boost irradiation in DCIS that have completed patient accrual. The TROG 07.01 trial (NCT00470236) endorsed by many international groups including IBCSG, BIG, NCIC and EORTC is a 4-arm trial. It studies both hypofractionation compared to standard fractionation and boost versus no boost in non-low-risk patients as determined by patient age $<50$ years or the presence of risk factors such as symptomatic presentation, palpable tumor, multifocal disease, microscopic tumor size $\geq 1.5 \mathrm{~cm}$ and intermediate or high nuclear grade, central necrosis or comedo histology in patients older than 50 years. The French Bonbis trial (NCT00907868) randomized patients to conventionally fractioned whole-breast irradiation with or without an additional tumor bed boost of $16 \mathrm{~Gy}$. LR is the primary endpoint for both trials.

\section{Hypofractionation}

Most prospective trials in DCIS patients used conventionally fractionated whole-breast RT up to a total dose of 48-50 Gy. Hypofractionation with single doses of 2.5-2.7 Gy up to total doses of 40-42.5 Gy have been extensively studied in invasive breast cancer. In the START A and B trials, there were no differences in terms of LR but late toxicity favored the hypofractionation arms [30]. Overall mortality, distant recurrence and late morbidity were significantly lower in patients randomly assigned to hypofractionation in the START B trial. Whelan et al. [31] reported the 10-year results of 1,234 patients randomized to either $50 \mathrm{~Gy}$ in 25 fractions or 42.5 Gy in 16 fractions. They showed non-inferiority of the hypofractionated regimen for LR. There was no significant difference for overall or disease-free survival.

In the SweDCIS trial, a subgroup of patients received hypofractionated RT (48 Gy in 20 fractions, 4 fractions/week) comparable to the RTOG 9804-trial (42.5 Gy in 16 fractions), no subgroup analyses according to the fractionation regimen were provided. Ciervide et al. [32] performed a subgroup analysis of 2 prospective trials on hypofractionation, including 145 patients with DCIS. Patients were treated with either 42 Gy in 15 fractions or 40.5 Gy in 15 fractions with a concomitant daily boost of 0.5 Gy to the resection cavity up to a total dose of $48 \mathrm{~Gy}$. After a median follow-up of 5 years, $4.1 \%$ of patients developed an LR. Toxicity was low, with $91 \%$ of patients having good-to-excellent cosmesis. A similar concept may also be feasible in invasive breast cancer [33].

In a multicenter retrospective study from Canada, Hathout et al. [34] observed a 5-year LR rate of around 3\% in 440 women using the Whelan regimen of $42.5 \mathrm{~Gy}$ delivered in 16 fractions. Lalani et al. [35] performed a population-based analysis of 1,609 patients from the Ontario Cancer Registry. Patients receiving hypofractionated RT (40-44 Gy in 16 fractions) were slightly older, had unifocal tumors more frequently and were more likely to receive a tumor bed boost. On univariate analysis, 5- and 10-year LR-free survival were significantly higher with hypofractionation than with conventional fractionation $(p=0.03)$. However, there was no significant difference on multivariate analysis.

A recent meta-analysis of Nilsson et al. [29] included 2,534 patients from 4 retrospective studies, and concluded that there was no significant difference between conventional and hypofractionated RT for DCIS; however, a trend for lower recurrence with hypofractionation was observed (odds rate $0.78, \mathrm{p}=0.08$ ). The quality of evidence from the included trials was rated as low.

In summary, hypofractionation seems to be a safe alternative for patients with DCIS undergoing BCS. However, underlying biases cannot be excluded and the results should be validated by further prospective trials.

\section{Discussion}

The optimal management of patients with DCIS after BCS remains controversial due to the different biological behavior of DCIS, the uncertainty of predicting LR risk individually and the absence of any survival benefit.

To reflect the role of RT in preventing LR after BCS in pure DCIS, the biologically as well as clinically important question that arises is whether the continuously increasing rate of LR over time after complete tumor resection (R0) and adjuvant RT represents either true LRs of the primary DCIS or, alternatively, constitutes second tumor manifestations. Long-term results from the SweDCIS [4] and the EORTC 10853 [36] trials have shown that the risk for LR is highest during the first 5 years after diagnosis with almost no non-invasive recurrences occurring beyond 10 years. The incidence of invasive LR, however, continues in a similar manner over time, independently of adjuvant RT, which implies that these LRs might be second tumor manifestations [4, 36]. New approaches such as molecular genetic analyses using multigene expression assays may help to clarify this clinically relevant issue. 
Considering the data from RCTs and meta-analyses, RT is beneficial in terms of prevention of in-breast recurrences and, thus, breast conservation. However, the increasing proportion of clinically inapparent tumors detected due to the introduction of mammography screening and the advances in pre- and postoperative imaging as well as pathology have to be kept in mind when interpreting these results [37].

Multiple attempts have been made to find clinically sufficient criteria for the selection of patients at low risk of recurrence. However, there is no safe and uniform definition for these criteria. There is a need to establish a consensus on which prognostic factors (clinical, molecular pathological, biomarkers) to use when selecting patients at low risk of recurrence who might qualify for BCS without adjuvant RT. The interdisciplinary discussion should incorporate clinical prognostic factors as well as comorbidities, benefit and harm caused by RT, and patient preferences into the decision-making process [38]. These prognostic markers, however, have to be validated in prospective trials.
Noteworthy, and a strong argument for considering RT, are the results of the RTOG 9804 trial demonstrating that even in patients with clinically determined low-risk DCIS, adjuvant RT significantly lowers the risk of LR [20].

Hypofractionated RT seems to be a valid treatment option for patients with DCIS, but further prospective studies are needed to define its role more precisely. The same is true for the application of a boost restricted to patients considered to be at high risk for an LR. There are also several ongoing trials assessing the role of accelerated partial breast irradiation in DCIS, but mature data are still pending.

\section{Disclosure Statement}

The authors declare no conflict of interest.

\section{References}

1 Lynge E, Ponti A, James T, et al.: Variation in detection of ductal carcinoma in situ during screening mammography: A survey within the international cancer screening network. Eur J Cancer 2014;50:185-192.

$\checkmark 2$ Souchon R, Sautter-Bihl ML, Sedlmayer F, et al.: DEGRO practical guidelines: Radiotherapy of breast cancer II: Radiotherapy of non-invasive neoplasia of the breast. Strahlenther Onkol 2014;190:8-16.

$\checkmark 3$ Early Breast Cancer Trialists' Collaborative Group, Correa C, McGale P, et al.: Overview of the randomized trials of radiotherapy in ductal carcinoma in situ of the breast. J Natl Cancer Inst Monogr 2010;2010:162-177.

4 Warnberg F, Garmo H, Emdin S, et al.: Effect of radiotherapy after breast-conserving surgery for ductal carcinoma in situ: 20 years follow-up in the randomized SweDCIS trial. J Clin Oncol 2014;32:3613-3618

5 Bekelman JE, Sylwestrzak G, Barron J, et al.: Uptake and costs of hypofractionated vs conventional whole breast irradiation after breast conserving surgery in the United States, 2008-2013. JAMA 2014;312:2542-2550.

6 Bartelink H, Maingon P, Poortmans P, et al.: Wholebreast irradiation with or without a boost for patients treated with breast-conserving surgery for early breast cancer: 20-year follow-up of a randomised phase 3 trial. Lancet Oncol 2015;16:47-56.

7 Silverstein MJ, Poller DN, Waisman JR, et al.: Prognostic classification of breast ductal carcinoma-in-situ. Lancet 1995;345:1154-1157.

8 Silverstein MJ, Lagios MD, Craig PH, et al.: A prognostic index for ductal carcinoma in situ of the breast. Cancer 1996;77:2267-2274.

9 Silverstein MJ: The University of Southern California/ Van Nuys Prognostic Index for ductal carcinoma in situ of the breast. Am J Surg 2003;186:337-343.

10 Boland GP, Chan KC, Knox WF, et al: Value of the Van Nuys Prognostic Index in prediction of recur rence of ductal carcinoma in situ after breast-conserving surgery. Br J Surg 2003;90:426-432.

11 MacAusland SG, Hepel JT, Chong FK, et al.: An attempt to independently verify the utility of the Van Nuys Prognostic Index for ductal carcinoma in situ. Cancer 2007;110:2648-2653.
12 Altintas S, Toussaint J, Durbecq V, et al.: Fine tuning of the Van Nuys prognostic index (VNPI) 2003 by integrating the genomic grade index (GGI): New tools for ductal carcinoma in situ (DCIS). Breast J 2011;17: 343-351.

13 Kerlikowske K, Molinaro AM, Gauthier ML, et al.: Biomarker expression and risk of subsequent tumors after initial ductal carcinoma in situ diagnosis. J Natl Cancer Inst 2010;102:627-637.

14 Solin LJ, Gray R, Baehner FL, et al.: A multigene expression assay to predict local recurrence risk for ductal carcinoma in situ of the breast. J Natl Cancer Inst 2013;105:701-710.

15 Hughes LL, Wang M, Page DL, et al.: Local excision alone without irradiation for ductal carcinoma in situ of the breast: A trial of the Eastern Cooperative Oncology Group. J Clin Oncol 2009;27:5319-5324.

16 Wong JS, Kaelin CM, Troyan SL, et al.: Prospective study of wide excision alone for ductal carcinoma in situ of the breast. J Clin Oncol 2006;24:1031-1036.

17 Wong JS, Chen YH, Gadd MA, et al.: Eight-year update of a prospective study of wide excision alone for small low- or intermediate-grade ductal carcinoma in situ (DCIS). Breast Cancer Res Treat 2014;143:343-350.

18 Williams KE, Barnes NL, Cramer A, et al.: Molecular phenotypes of DCIS predict overall and invasive recurrence. Ann Oncol 2015;26:1019-1025.

19 Curigliano G, Disalvatore D, Esposito A, et al.: Risk of subsequent in situ and invasive breast cancer in human epidermal growth factor receptor 2-positive ductal carcinoma in situ. Ann Oncol 2015;26:682-693.

20 McCormick B, Winter K, Hudis C, et al.: RTOG 9804: A prospective randomized trial for good-risk ductal carcinoma in situ comparing radiotherapy with observation. J Clin Oncol 2015;33:709-715.

21 Sedlmayer F, Sautter-Bihl ML, Budach W, et al.: DEGRO practical guidelines: Radiotherapy of breast cancer I: Radiotherapy following breast conserving therapy for invasive breast cancer. Strahlenther Onkol 2013;189:825-833.

22 Duma MN, Molls M, Trott KR: From heart to heart for breast cancer patients - cardiovascular toxicities in breast cancer radiotherapy. Strahlenther Onkol 2014;190:5-7.
23 Boekel NB, Schaapveld M, Gietema JA, et al.: Cardiovascular morbidity and mortality after treatment for ductal carcinoma in situ of the breast. J Natl Cancer Inst 2014;106,pii:dju 156.

24 Julian TB, Mamounas EP, Costantino JP, Wolmark N: Is boost therapy necessary in the treatment of DCIS? J Clin Oncol 2008;26:537.

25 Omlin A, Amichetti M, Azria D, et al.: Boost radiotherapy in young women with ductal carcinoma in situ: A multicentre, retrospective study of the rare cancer network. Lancet Oncol 2006;7:652-656.

26 Wong P, Lambert C, Agnihotram RV, et al.: Ductal carcinoma in situ-the influence of the radiotherapy boost on local control. Int J Radiat Oncol Biol Phys 2012;82:e153-158.

27 Meattini I, Livi L, Franceschini D, et al.: Role of radiotherapy boost in women with ductal carcinoma in situ: A single-center experience in a series of 389 patients. Eur J Surg Oncol 2013;39:613-618.

28 Monteau A, Sigal-Zafrani B, Kirova YM, et al.: Ductal carcinoma in situ of the breast with close or focally involved margins following breast-conserving surgery: Treatment with reexcision or radiotherapy with increased dosage. Int J Radiat Oncol Biol Phys 2009;75: 1021-1028.

29 Nilsson C, Valachis A: The role of boost and hypofractionation as adjuvant radiotherapy in patients with DCIS: A meta-analysis of observational studies. Radiother Oncol 2015;114:50-55.

30 Haviland JS, Owen JR, Dewar JA, et al.: The UK standardisation of breast radiotherapy (START) trials of radiotherapy hypofractionation for treatment of early breast cancer: 10-year follow-up results of two randomised controlled trials. Lancet Oncol 2013;14:10861094.

31 Whelan TJ, Pignol JP, Levine MN, et al.: Long-term results of hypofractionated radiation therapy for breast cancer. N Engl J Med 2010;362:513-520.

32 Ciervide R, Dhage S, Guth A, et al.: Five year outcome of 145 patients with ductal carcinoma in situ (DCIS) after accelerated breast radiotherapy. Int J Radiat Oncol Biol Phys 2012;83:e159-164. 
33 Dellas K, Vonthein R, Zimmer J, et al.: Hypofractionation with simultaneous integrated boost for early breast cancer: Results of the German multicenter phase II trial (ARO-2010-01). Strahlenther Onkol 2014;190 646-653.

34 Hathout L, Hijal T, Theberge V, et al.: Hypofractionated radiation therapy for breast ductal carcinoma in situ. Int J Radiat Oncol Biol Phys 2013;87:1058-1063.
35 Lalani N, Paszat L, Sutradhar R, et al.: Long-term outcomes of hypofractionation versus conventional radiation therapy after breast-conserving surgery for ductal carcinoma in situ of the breast. Int J Radiat Oncol Biol Phys 2014;90:1017-1024.

36 Donker M, Litiere S, Werutsky G, et al.: Breast-conserving treatment with or without radiotherapy in ductal carcinoma in situ: 15-year recurrence rates and outcome after a recurrence, from the EORTC 10853 randomized phase III trial. J Clin Oncol 2013;31:4054-4059.
37 Recht A: Are the randomized trials of radiation therapy for ductal carcinoma in situ still relevant? J Clin Oncol 2014;32:3588-3590.

38 Smith BD: When is good enough really good enough? Defining the role of radiation in low-risk ductal carcinoma in situ. J Clin Oncol 2015;33:686-691. 